L. Bădescu

Nagoya Math. J.

Vol. 61 (1976), 205-220

\title{
ON CERTAIN ISOLATED NORMAL SINGULARITIES
}

\author{
LUCIAN BǍDESCU
}

\section{Introduction.}

In the following we shall fix an algebraically closed field $K$ of arbitrary characteristic. The term variety will mean an algebraic scheme over $K$ which is integral. In general we shall use the notations and the terminology of Éléments de Géométrie Algébrique of A. Grothendieck and J. Dieudonné. For instance, if $Z$ is a variety and $x \in Z$ is a point, then $O_{Z, x}$ means the local ring of $Z$ at $x$ and $m_{x}$ - the maximal ideal of $O_{Z, x}$.

Let $Y$ be a variety and $y \in Y$ a closed normal point, which is defined as reduced closed subscheme of $Y$ by the coherent sheaf of ideals $J$, where $J_{y}=m_{y}$ and $J_{z}=O_{Y, z}$ for every $z \neq y$. Consider the cartesian diagram of blowing up of $Y$ of center $J$ :

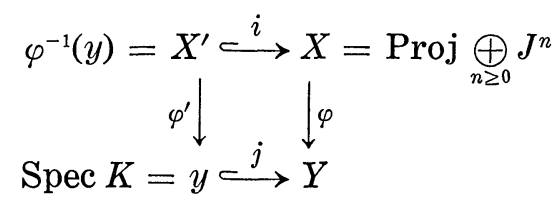

in which the exceptional locus $X^{\prime}$ is defined as closed subscheme of $X$ by the coherent (invertible) ideal $I=O_{X}(1)$. If $X^{\prime}$ is non singular, then $X$ is also non singular at each point of $X^{\prime}$, because the ideal $I$ is invertible. Thus the point $y$ is either non singular or an isolated normal singularity of $Y$.

The aim of this paper is the study of normal (isolated) singularities which give rise by blowing up to a diagram (*) in which $X^{\prime}$ is isomorphic to a product $P^{n_{1}} \times \cdots \times P^{n_{r}}$ of $r(r \geqslant 1)$ projective spaces over $K$ $\left(n_{1}, \cdots, n_{r}\right.$ being strictly positive integers). Because in this case the Picard group of $X^{\prime}$ is $Z^{r}$, then the conormal bundle (or sheaf - we do not make any distinction between these two terms) of $X^{\prime}$ into $X$ is of the

Received July 22, 1974. 
form $i^{*} I=p_{1}^{*}\left(O\left(s_{1}\right)\right) \otimes \cdots \otimes p_{r}^{*}\left(O\left(s_{r}\right)\right.$ ) (in the following denoted simply by $O\left(s_{1}, \cdots, s_{r}\right)$ ), where $p_{v}$ is the $\nu^{\text {th }}$ canonical projection of $P^{n_{1}} \times \cdots \times P^{n_{r}}$ and $O\left(s_{\nu}\right)$ is the invertible sheaf on $P^{n_{\nu}}$ associated to the hypersurfaces of degree $s_{\nu}$. Because $i^{*} I$ is ample on $X^{\prime}$, it follows that all $s_{\nu}$ are strictly positive integers. Thus it is convenient to give:

DEFINITION. In the above situation, we shall say that the point $y$ is an isolated normal singularity of type $\left(\left(n_{1}, s_{1}\right), \ldots,\left(n_{r}, s_{r}\right)\right)$, with all $n_{\nu}, s_{\nu}$ strictly positive integers.

In particular the dimension of such a singularity is $n_{1}+\cdots+n_{r}+1$.

In the section 1 the main result (theorem 1 ) asserts that any two singularities of the same type $\left(\left(n_{1}, s_{1}\right), \cdots,\left(n_{r}, s_{r}\right)\right)$ are analytically equivalent in the sense that their completions in the topologies of their maximal ideals are isomorphic. For the case where the ground field $K$ is the field of the complex numbers, this theorem follows from an analytical result of Grauert (see H. Grauert-Über Modifikationen und exzeptionelle analytische Mengen, Math. Annalen, 146 (1962), pp. 331-368, § 4, theorem 7). Our proof is purely algebraic and uses some facts from Lefschetz's theory in Grothendieck's form (see [10]), so that the result holds in every characteristic. As a corollary, we prove that these singularities are always Cohen-Macaulay. Finally one computes the multiplicity and the divisor class group of the local ring of such a singularity. All results of section 1 extend some of a previous paper (see [7]), where one considered only the case $r=1$ (which arises in the context of "rational contractions" in the sense of [5]).

In the second part of the paper we are interested in the behaviour of the dualizing sheaf $\omega_{Y}$ (see [1] for the definition) by taking the inverse image via the morphism $\varphi$ of blowing up, and in particular when $\omega_{Y}$ is invertible. The main result is theorem 2 which can be stated as follows: let $y \in Y$ be an isolated singularity of type $\left(\left(n_{1}, s_{1}\right), \ldots,\left(n_{r}, s_{r}\right)\right)$. Then its local ring $O_{Y, y}$ is Gorenstein if and only if $1+n_{1}=a s_{1}, \cdots, 1+$ $n_{r}=a s_{r}$ where $a$ is a (necessarily positive) integer, and in this case $\omega_{Y}$ is invertible in a neighbourhood of $y$ and $\omega_{X}=\varphi^{*}\left(\omega_{Y}\right) \otimes I^{1-a}$.

In particular, if $a=1$, one get the formula $\omega_{X}=\varphi^{*}\left(\omega_{Y}\right)$; in other words the isolated normal singularities $y \in Y$ of type $\left(\left(n_{1}, n_{1}+1\right)\right.$, $\cdots,\left(n_{r}, n_{r}+1\right)$ ) have the property that the canonical divisor of $Y$ is locally $\leftrightarrow$ principal at $y$ and its inverse image by $\varphi$ is a canonical divisor of $X$. M. Artin proved that a rational singularity of a surfaces $Y$ (see 
[3] for the definition of the rational singularities of surfaces) has this last property to respect to a minimal desingularization $\varphi: X \rightarrow Y$ if and only if it is a rational double point (see [2]).

As a consequence of theorem 2, one proves among others, that the vertex of the affine cone of $P^{n} \times P^{m}$ via the Segre imbedding is a Gorenstein point if and only if $n=m$.

\section{§1. An example and the analytical equivalence of any two singularities of the same type $\left(\left(n_{1}, s_{1}\right), \cdots,\left(n_{r}, s_{r}\right)\right)$.}

We begin with a standard example of isolated normal singularity of type $\left(\left(n_{1}, s_{1}\right), \cdots,\left(n_{r}, s_{r}\right)\right)$.

EXAMPLE. Let us fix $2 r(r \geqslant 1)$ strictly positive integers $n_{1}, \cdots, n_{r}$; $s_{1}, \cdots, s_{r}$ and put $X^{\prime}=P^{n_{1}} \times \cdots \times P^{n_{r}}$. Denote by $L$ the invertible sheaf $O\left(s_{1}, \cdots, s_{r}\right)$ as above and consider the imbedding $\lambda: X^{\prime} \subset P^{t}$ (with $\left.t=\left(\begin{array}{c}n_{1}+s_{1} \\ n_{1}\end{array}\right) \cdots\left(\begin{array}{c}n_{r}+s_{r} \\ n_{r}\end{array}\right)-1\right)$ given by equations :

$$
T_{i_{10}, i_{11}, \cdots, i_{1 n_{1}}, \cdots ; i_{r 0}, i_{r 1}, \cdots, i_{r n_{r}}}=u_{10}^{i_{10}} \cdots u_{1_{1_{1}}}^{i_{1 n}} \cdots u_{r 0}^{i_{r 0}} \cdots u_{r n_{r}}^{i_{r n}}
$$

where $u_{j 0}, \cdots u_{j n_{j}}$ are the homogeneous coordinates of the projective space $P^{n_{j}}, i_{j h} \geqslant 0$ and $i_{j 0}+\cdots+i_{j n_{j}}=s_{j}$. It is not difficult to see that the parametric equations (1) are equivalent with the implicites ones:

$$
T_{\ldots ; i_{j 0}, i_{j 1}, \ldots, i_{j n_{j}} ; \ldots} T_{\ldots ; h_{j 0}, h_{j 1}, \ldots, h_{j n_{j}} ; \ldots}=T_{\ldots ; k_{j 0}, k_{j 1} ; \ldots, k_{j n_{j}} ; \ldots} T_{\ldots ; e_{j 0}, e_{j 1}, \ldots, e_{j n_{j}} ; \cdots}
$$

where all the indexes are non negative, $i_{j 0}+\cdots+i_{j n_{j}}=h_{j 0}+\cdots+$ $h_{j n_{j}}=k_{j 0}+\cdots+k_{j n_{j}}=e_{j 0}+\cdots+e_{j n_{j}}=s_{j}$ for all $j=1,2, \cdots, r$ and $i_{j f}+h_{j f}=k_{j f}+e_{j f}$ for all $j=1,2, \cdots, r$ and $f=0,1, \cdots, n_{j}$.

If $s_{1}=\cdots=s_{r}=1, \lambda$ is the Segre immersion, while the case $r=1$ and $s=s_{1}>0$ gives rise to the Veronese immersion of $P^{n}$ by forms of degree $s$. In general we shall say that the imbedding $\lambda$ is the SegreVeronese immersion.

Now the vertex of the affine cone of $X^{\prime}$ via the imbedding $\lambda$ is an isolated normal singularity of type $\left(\left(n_{1}, s_{1}\right), \cdots,\left(n_{r}, s_{r}\right)\right)$. In fact the blowing up of this cone at its vertex coincides with the vector bundle $V(L)$ associated to the invertible sheaf $L$ and the immersion of the exceptional locus $X^{\prime}$ into $V(L)$-with the zero section of this bundle (see EGA II, § 8). 
Proposition 1. Let $y \in Y$ be an isolated normal singularity of type $\left(\left(n_{1}, s_{1}\right), \cdots,\left(n_{r}, s_{r}\right)\right)$. With the notations of the diagram $(*)$ from introduction, one has: $R^{q} \varphi_{*}\left(I^{n}\right)=0$ for $q>0$ and $n \geqslant 0, J^{n}=\varphi_{*}\left(I^{n}\right)$ for $n \geqslant 0$ and there exists a canonical isomorphism of $K$-algebras

$$
\alpha: \bigoplus_{n \geq 0} m_{y}^{n} / m_{y}^{n+1} \longrightarrow \underset{n \geq 0}{\bigoplus} H^{0}\left(X^{\prime}, i^{*} I^{n}\right) .
$$

The proof of this proposition is standard and just an adaptation of the proof of theorem 2 of [6], once one knows how to compute the cohomology of $i^{*} I^{n}$. But this is not difficult using Kunneth's formulas:

$$
H^{q}\left(X^{\prime}, i^{*} I^{n}\right)=\sum_{q_{1}+\cdots+q_{r}=q_{,} q_{i} \geq 0} H^{q_{1}}\left(P^{n_{1}}, O\left(n s_{1}\right)\right) \otimes \cdots \otimes H^{q_{r}}\left(P^{n_{r}}, O\left(s n_{r}\right)\right) .
$$

Because the cohomology of invertible sheaves on a projective space is known (see FAC or EGA III), one get: $H^{q}\left(X^{\prime}, i^{*} I^{n}\right)=0$ for all $q>0$ and $n \geqslant 0$ and

$$
\operatorname{dim}_{K} H^{0}\left(X^{\prime}, i^{*} I^{n}\right)=\left(\begin{array}{c}
n_{1}+s_{1} n \\
n_{1}
\end{array}\right) \cdots\left(\begin{array}{c}
n_{r}+s_{r} n \\
n_{r}
\end{array}\right) .
$$

COROLLARY 1. In the hypothesis of proposition 1, the dimension of the Zariski tangent space of $Y$ at $y$ is $\left(\begin{array}{c}n_{1}+s_{1} \\ n_{1}\end{array}\right) \cdots\left(\begin{array}{c}n_{r}+s_{r} \\ n_{r}\end{array}\right)$ and the multiplicity of the local ring $O_{Y, y}$ is $\frac{\left(n_{1}+\cdots+n_{r}\right) !}{n_{1} ! \cdots n_{r} !} s_{1}^{n_{1}} \cdots s_{r}^{n_{r}}$.

Proof. All is clear because the formula (4) and proposition 1 give the Samuel function and one applies the definition of the multiplicity.

COROLLARY 2. In the hypothesis of proposition 1, if $Y$ is proper over $K$, then $\chi\left(Y, O_{Y}\right)=\chi\left(X, O_{X}\right)$.

Proof. Consider the Leray spectral sequence:

$$
E_{2}^{p q}=H^{p}\left(Y, R^{q} \varphi_{*} O_{X}\right) \Longrightarrow H^{p+q}\left(X, O_{X}\right) \text {. }
$$

The proposition 1 says that this spectral sequence degenerates, therefore for all $p \geqslant 0$ one get the isomorphisms $H^{p}\left(Y, \varphi_{*} O_{X}\right)=H^{p}\left(X, O_{X}\right)$ and finally $\chi\left(Y, O_{Y}\right)=\chi\left(X, O_{X}\right)$ because $\varphi_{*} O_{X}=O_{Y}$.

Remarks 1) The formula (3) shows that $H^{q}\left(X^{\prime}, O\left(a_{1}, \cdots, a_{r}\right)\right)=0$ for $0<q<n_{1}+\cdots+n_{r}$ and for every $a=\left(a_{1}, \cdots, a_{r}\right) \in Z^{r}$ and $H^{q}\left(X^{\prime}, O\left(a_{1}\right.\right.$, $\left.\left.\cdots, a_{r}\right)\right)=0$ for $q=n_{1}+\cdots+n_{r}$ and for every $a$ such that $a_{1} \geqslant-n_{1}$, $\cdots, a_{r} \geqslant-n_{r}$. 
2) There is a $1-1$ correspondence between the Picard group of $Y$ and the subgroup of the Picard group of $X$ consisting by the classes of isomorphic invertible sheaves $L$ on $X$ for which there exists an open neighbourhood $U$ of $X^{\prime}$ in $X$ (and depending of $L$ ) such that $L / U \cong O_{X} / U$. The correspondence is given by $\varphi^{*}$ and $\varphi_{*}$. Indeed, one applies the fact that $\varphi_{*} O_{X} \cong O_{Y}$ (proposition 1). Moreover, if $L$ is an invertible sheaf on $X$, trivial in a neighbourhood of $X^{\prime}$, then $R^{q} \varphi_{*}(L)=0$ for every $q>0$. Indeed, because $X-X^{\prime}=Y-y$, one has only to look at a suitable open neighbourhood of $X^{\prime}$ in $X$ in which $L$ is trivial and the assertion becomes $R^{q} \varphi_{*}\left(O_{X}\right)=0$ for every $q>0$, which is contained in the proposition 1.

Now we arrive at the main result of this section:

THEOREM 1. Let $(Y, y)$ and $(\tilde{Y}, \tilde{y})$ be two isolated normal singularities of the same type $\left(\left(n_{1}, s_{1}\right), \cdots,\left(n_{r}, s_{r}\right)\right)$. Then the completions of the local ring $O_{Y, y}$ and $O_{\widetilde{Y}, \tilde{y}}$ in the topologies of their maximal ideals $m_{y}$ and $m_{\tilde{y}}$, are isomorphic.*)

Proof. It is obviously sufficient to take for $(\tilde{Y}, \tilde{y})$ the singularity considered in the above example. Let us denote by $A$ the $m_{y}$-adic completion of the local ring $O_{Y, y}$ and by $m$ the maximal ideal of $A$. Consider the cartesian diagram of blowing up of $\operatorname{Spec}(A)$ (denoted again by $Y$ ) of center $m$ :

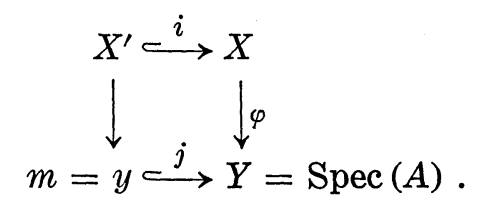

Since $X^{\prime}=\operatorname{Proj} \underset{n \geq 0}{\bigoplus_{n}} m^{n} / m^{n+1}$ and $m^{n} / m^{n+1}=m_{y}^{n} / m_{y}^{n+1}$, one deduces that $X^{\prime}$ is again isomorphic to $P^{n_{1}} \times \cdots \times P^{n_{r}}$ and $i^{*} I=O\left(s_{1}, \cdots, s_{r}\right)$.

Step 1. The natural homomorphism of restriction $i^{*}: \operatorname{Pic}(X) \rightarrow$ $\operatorname{Pic}\left(X^{\prime}\right)=Z^{r}$ is an isomorphism.

In fact the pair $\left(X, X^{\prime}\right)$ verifies the effective Lefschetz condition (see [10] éxposé $X$ ). In other words the functor $F \rightarrow \hat{F}$ defined on the category of the locally free sheaves on $X$ of finite rank into the category of locally free sheaves of finite rank on the formal completion $\hat{X}$ of $X$ along $X^{\prime}$ is an equivalence of categories. This follows from the comparison theorems

\footnotetext{
*) See added in proof.
} 
in algebraic geometry (EGA III (4.1.5) and (5.1.4)). More precisely, the first theorem of comparison asserts that this functor is fully faithfull. On the other hand, we have to observe that the single open neighbourhood of $X^{\prime}$ in $X$ is $X$ itself ; then the second theorem of comparison says that this functor is "surjective", i.e. for every coherent locally free sheaf $G$ over $\hat{X}$ there exists a coherent sheaf over $X$ such that $\hat{F}=G$. With the remark just made and the fact that the morphism $\hat{X} \rightarrow X$ is flat, one can easily deduce that $F$ is also locally free.

Now, in order to deduce that the homomorphism $i^{*}$ is anis omorphism, the proposition 3.12 from [10] éxposé XI reduces the problem to verify that $H^{q}\left(X^{\prime}, i^{*} I^{n}\right)=0$ for all $n \geqslant 0$ and $q=1,2$. But this condition is clearly fullfiled because $X^{\prime}$ is a product of projective spaces and $i^{*} I^{n}=O\left(n s_{1}\right.$, $\left.\cdots, n s_{r}\right)$.

Step 2. For every $i=1,2, \cdots r$ there exists an invertible sheaf $L_{i}$ on $X$ such that $i^{*}\left(L_{i}\right)=O(0, \cdots, 0,1,0, \cdots, 0)$ (1 on $i^{\text {th }}$ place $)$ and $H^{1}\left(X, L_{i} \otimes I\right)=0$.

Indeed, the existence of $L_{i}$ (for all $i$ ) is given by step 1 , so we have only to verify that $H^{1}\left(X, L_{i} \otimes I\right)=0$; for, consider the exact sequence:

$$
0 \rightarrow L_{i} \otimes I^{n+1} \rightarrow L_{i} \otimes I^{n} \rightarrow i_{*} O\left(s_{1}, \cdots, s_{i-1}, s_{i}+1, s_{i+1}, \cdots, s_{r}\right)=N_{i} \rightarrow 0
$$

which induces the exact sequence of cohomology:

$$
H^{1}\left(X, L_{i} \otimes I^{n+1}\right) \rightarrow H^{1}\left(X, L_{i} \otimes I^{n}\right) \rightarrow H^{1}\left(X^{\prime}, N_{i}\right)=0
$$

and the assertion follows because in any case $H^{1}\left(X, L_{i} \otimes I^{n}\right)=0$ for $n \gg 0$ and one applies descending induction on $n$.

Step 3. Construction of an isomorphism between $A$ and $\hat{O}_{\tilde{Y}, \tilde{y}}\left(m_{\tilde{y}^{-}}\right.$ adic completion of the local ring $O_{\widetilde{Y}, \tilde{y}}$ of the vertex of the cone of $X^{\prime}$ via the Segre-Veronese imbedding).

First of all, by construction of $L_{i}$, we have the exact sequence

$$
0 \rightarrow L_{i} \otimes I \rightarrow L_{i} \rightarrow i_{*} O(0, \cdots, 0,1,0, \cdots, 0) \rightarrow 0
$$

which gives rise to the exact sequence of cohomology:

$$
H^{0}\left(X, L_{i}\right) \rightarrow H^{0}\left(P^{n_{i}}, O(1)\right) \rightarrow H^{1}\left(X, L_{i} \otimes I\right)=0 \text {. }
$$

If $u_{i 0}^{\prime}, \cdots, u_{i n_{i}}^{\prime}$ is a base of the $K$-vector space $H^{0}\left(X^{\prime}, O(0, \cdots, 0,1,0\right.$, $\cdots, 0))=H^{0}\left(P^{n_{i}}, O(1)\right)$, it follows that there exist $u_{i 0}, \cdots, u_{i n_{i}} \in H^{0}\left(X, L_{i}\right)$ such that the restriction of $u_{i j}$ to $X^{\prime}$ is precisely $u_{i j}^{\prime}$. Therefore one can 
consider the following sections of $I \cong L_{1}^{s_{1}} \otimes \cdots \otimes L_{r}^{s_{r}}$ (they are certainly isomorphic because they have the same restrictions to $X^{\prime}$ and by step 1 , $i^{*}$ is bijective) :

$$
a_{i_{10}, \cdots, i_{1 n_{1}}, \cdots ; i_{r 0}, \cdots, n_{r}}=u_{10}^{i_{10}} \cdots u_{1 n_{1}}^{i_{1 n_{1}}} \cdots u_{r 0}^{i_{r 0}} \cdots u_{r n_{r}}^{i_{r n_{r}}} \in H^{0}(X, I)
$$

where $i_{j k} \geqslant 0$ and $i_{j 0}+\cdots+i_{j n_{j}}=s_{j}$. But $H^{0}(X, I)=m$ (proposition 1) and these sections satisfy Segre-Veronese equations (2). By Nakayama one deduces that they form a system of generators of the maximal ideal $m$. Because the ground field $K$ is contained in $A$, we can therefore construct a homomorphism of $K$-algebras:

$$
\varepsilon: K\left[\cdots, T_{;} \cdots ; i_{j 0}, \cdots, i_{j_{j}} ; \cdots, \cdots\right] / \mathfrak{U} \rightarrow A
$$

where $\mathfrak{A}$ is the ideal generated by equations (2), such that $T_{\ldots ; i_{0}, \ldots, i_{j j_{j}} ; \ldots}$ is mapped into $u_{\ldots ; i_{j 0}, \ldots, i_{j n_{j}} ; \ldots}$. Hence one get a local homomorphism $\bar{\varepsilon}: \hat{O}_{\widetilde{Y}, \tilde{y}} \rightarrow A$ with the property $\bar{\varepsilon}\left(\hat{m}_{\tilde{y}}\right)=m$. Now it is clear that $\bar{\varepsilon}$ is an isomorphism, because the residue fields of both rings are the same (namely $K)$, which completes the proof of theorem 1 .

COROLlaRY 1. Let $y \in Y$ be an isolated normal singularity of type $\left(\left(n_{1}, s_{1}\right), \cdots,\left(n_{r}, s_{r}\right)\right)$; then the local ring $O_{Y, y}$ is Cohen-Macaulay.

Proof. $O_{Y, y}$ is Cohen-Macaulay if and only if its $m_{y}$-adic completion is so, therefore theorem 1 reduces the problem to see that the local ring of the isolated normal singularity considered in the example is Cohen-Macaulay. Now one can prove this using the following criterium ([12], proposition 4, appendix of the lecture 13):

(***) Let $R$ be a finitely generated graded algebra such that $R_{0}=K$ is a field and $R_{n}$ is generated by $R_{1}^{\otimes n}$. Let $X^{\prime}=\operatorname{Proj}(R)$ and $R_{m}$ be the localization of $R$ to respect of the maximal ideal $m=\bigoplus_{n \geq 1} R_{n}$. Then $\operatorname{depth}\left(R_{m}\right) \geqslant k$ (where $k \geqslant 2$ ) if and only if the canonical homomorphism of graded algebras

$$
\alpha: R \longrightarrow \bigoplus_{n \in \boldsymbol{Z}} H^{0}(X ; O(n))
$$

is an isomorphism and $H^{i}\left(X^{\prime}, O(n)\right)=0$ for all $n$ and $1 \leqslant i \leqslant k-2$.

In our case $R$ is the homogeneous coordinates ring of $X^{\prime}=P^{n_{1}} \times$ $\cdots \times P^{n_{r}}$ via the immersion $\lambda$ and $R_{m}=O_{\widetilde{Y}, \tilde{y}}$. The remark 1 and the fact that $X^{\prime}$ is projectively normal show that depth $\left(O_{\widetilde{Y}, \tilde{y}}\right) \geqslant n_{1}+\cdots+$ $n_{r}+1$ and therefore $O_{Y, y}$ is Cohen-Macaulay because $\operatorname{dim} O_{\widetilde{Y}, \tilde{y}}=n_{1}+$ $\cdots+n_{r}+1$. 
COROLlaRY 2. Let $y \in Y$ be an isolated normal singularity of type $\left(\left(n_{1}, s_{1}\right), \cdots,\left(n_{r}, s_{r}\right)\right)$ and $\hat{O}_{Y, y}$ the $m_{y}$-adic completion of its local ring $O_{Y, y}$. Then the divisor class group of $\hat{O}_{Y, y}$ (see N. Bourbaki, Algèbre commutative, chap. VII) is isomorphic to $\left.Z\right|_{d Z} \oplus Z^{r-1}$ where $d$ is the greatest common divisor of $s_{1}, \cdots, s_{r}$.

Proof. Put $A=\hat{O}_{Y, y}$ and consider the diagram of blowing up (**) from the proof of theorem 1. Because $X$ is non singular, it follows that the natural homomorphism of restriction $h^{*}: \operatorname{Pic}(X) \rightarrow \operatorname{Pic}\left(X-X^{\prime}\right)$ (where $h: X-X^{\prime} \smile X$ is the canonical open immersion) is surjective and $\operatorname{Ker}\left(h^{*}\right)$ is the cyclic (infinite) group generated by the class of $I$. Hence Pic $\left(X-X^{\prime}\right)$ is isomorphic to $Z^{r} /{ }_{\left(S_{1}, \cdots, s_{r}\right)} Z \cong Z /_{d Z} \oplus Z^{r-1}$ (because $i^{*}$ is an isomorphism, $\operatorname{Pic}\left(X^{\prime}\right)=Z^{r}$ and $i^{*} I=O\left(s_{1}, \cdots, s_{r}\right)$ ). On the other hand $\operatorname{Pic}\left(X-X^{\prime}\right)=\operatorname{Pic}(Y-y)$ is isomorphic to the divisor class group of the local ring $A$, because $\operatorname{dim}(A) \geqslant 2$.

§2. The dualizing sheaf and the isolated normal singularities of type $\left(\left(n_{1}, s_{1}\right)\right.$, $\left.\cdots,\left(n_{r}, s_{r}\right)\right)$.

First of all some terminology: a variety $Z$ is said to be CohenMacaulay (resp. Gorenstein) if for every point $x \in Z$, the local ring $O_{Z, x}$ is Cohen-Macaulay (resp. Gorenstein).

Now recall the central result about duality of $\leftrightarrow$ the projective Cohen-Macaulay varieties (see [1]):

THEOREM A (Grothendieck-Serre). Let $X$ be a projective CohenMacaulay variety of dimension $d$. Then there exists a uniquely determinated coherent $O_{X}$ module $\omega_{X}$ such that for all coherent $O_{X}$-modules $F$ and all $0 \leqslant p \leqslant d$ there exists a non singular pairing

$$
H^{p}(X, F) \times \operatorname{Ext}_{O_{X}}^{d-p}\left(F, \omega_{X}\right) \rightarrow K
$$

i.e. a natural isomorphism of $H^{p}(X, F)$ with the dual of $\operatorname{Ext}_{o_{X}}^{d-p}\left(F, \omega_{X}\right)$. If $F$ is locally free of rank $t$, then

$$
\operatorname{Ext}_{o_{X}}^{d-p}\left(F, \omega_{X}\right)=H^{d-p}\left(X, \check{F} \otimes \omega_{X}\right)
$$

where $\check{F}$ stands for the dual of $F . \omega_{X}$ can be constructed as follows: take an arbitrary imbedding $X \longrightarrow P^{n}=P$ and put $\omega_{X}=\operatorname{Ext}_{o_{P}}^{n-d}\left(O_{X}, O_{P}(-n-1)\right)$. If $X$ is non singular, then $\omega_{X}$ coincides to the (invertible) sheaf of germs of differential forms $\Omega_{X / K}^{d}$ of degree $d$. 
The following theorem of Grothendieck-Serre explains the importance of $\leftrightarrow$ Gorenstein varieties.

THeOREM B (see [4], proposition 5.1). If $X$ is a projective, CohenMacaulay variety then $\omega_{X}$ is invertible if and only if $X$ is Gorenstein (and in this case $\omega_{X}$ is said to be the canonical class of $X$ ).

Proposition 2. Let $Y$ be a variety and $y \in Y$ be a closed normal point. Consider the cartesian diagram (*) of blowing up of $Y$ of center $y$ (see introduction) and suppose $X^{\prime}$ non singular. Then, if $Y$ is Gorenstein at $y$, then the class of $\omega_{X^{\prime}}\left(\right.$ in Pic $\left.\left(X^{\prime}\right)\right)$ belongs to the $\leftrightarrow s u b$ group of Pic $\left(X^{\prime}\right)$ generated by the class of the conormal bundle of $X^{\prime}$ in $X$.

Proof. As in introduction, $y$ is an isolated singularity and $X^{\prime}$ is contained in the open set $\operatorname{Reg}(X)=U$ of non singular points of $X$. Define the invertible sheaf $L$ on $X$ such that $L / U=\left(\varphi^{*}\left(\omega_{Y}\right) / U\right)^{-1} \otimes\left(\omega_{X} / U\right)$ (indeed $\omega_{Y}$ is invertible in $\varphi(U)$ because $\varphi(U)$ is non singular outside of $y$ and Gorenstein at $y$ ) and $L / X-X^{\prime} \cong O_{X} / X-X^{\prime}$. Because $X^{\prime}$ is irreducible and $L$ is by construction trivial outside of $X^{\prime}$, it follows that $L \cong I^{c}$ where $c$ is an integer. Indeed, it is sufficient to prove that $L / U \cong I^{c} / U$, therefore to suppose $X$ non singular. Then let $D$ be a Weil divisor such that its associated invertible sheaf is $L$. Because $L / X-X^{\prime}$ is trivial, there exists a rational function $f$ on $X$ such that $\operatorname{div}_{X}(f) / X-$ $X^{\prime}=D / X-X^{\prime} . \quad$ Set $\operatorname{div}_{X}(f)=n X^{\prime}+\sum_{i} n_{i} D_{i}$ with $n, n_{i}$ integers and $D_{i}$ irreducible hypersurfaces distinct from $X^{\prime}\left(X^{\prime}\right.$ is irreducible because it is non singular and, on the other hand, it is connected taking into account of the normality of $y$ and the connectedness theorem of Zariski). Then $\operatorname{div}_{X}(f) / X-X^{\prime}=\sum_{i} n_{i} D_{i} / X-X^{\prime}$. Then $D=\sum_{i} n_{i} D_{i}+n^{\prime} X^{\prime}$, therefore $D=\operatorname{div}_{X}(f)+\left(n^{\prime}-n\right) X^{\prime}$ and take $c=n-n^{\prime}$. In particular, the isomorphism $L \cong I^{c}$ implies $i^{*}(L) \cong i^{*}\left(I^{c}\right)$, or, what is the same

$$
i^{*}\left(\omega_{X}\right) \cong\left(i^{*}(I)\right)^{c}
$$

(because $\left.i^{*} \varphi^{*}\left(\omega_{Y}\right) \cong O_{X^{\prime}}\right)$. Now it is well known that $i^{*}\left(\omega_{X}\right) \cong \omega_{X^{\prime}} \otimes i^{*}(I)$ (see [1] proposition 2.4, chap. I). Substituting this last formula in (5), one get:

$$
\omega_{X^{\prime}} \cong\left(i^{*}(I)\right)^{c-1} \text { and } \omega_{X} \cong \varphi^{*}\left(\omega_{Y}\right) \otimes I^{c}
$$

Q.E.D. 
COROLLARY 1. Let $y \in Y$ be an isolated normal singularity of type $\left(\left(n_{1}, s_{1}\right), \cdots,\left(n_{r}, s_{r}\right)\right)$. If its local ring $O_{Y, y}$ is Gorenstein, then

$$
\frac{n_{1}+1}{s_{1}}=\cdots=\frac{n_{r}+1}{s_{r}}=a \quad \text { with } a \in Z a \text { (positive) integer }
$$

and

$$
\omega_{X} \cong \varphi^{*}\left(\omega_{Y}\right) \otimes I^{1-a}
$$

Proof. In our case $i^{*}(I)=O\left(s_{1}, \cdots, s_{r}\right)$ and $\omega_{X^{\prime}}=O\left(-n_{1}-1, \cdots,-n_{r}\right.$ -1 ) because $X^{\prime}$ is a product of projective spaces. The corollary 1 follows now from (6), taking $a=1-c$.

COROLlARY 2. Let $X^{\prime}$ be a projective non singular variety imbedded in the projective space $P^{n}$. If the vertex of the affine cone of $X^{\prime}$ via this imbedding is Gorenstein, then there exists an integer $m \in Z$ such that $\omega_{X^{\prime}}=O_{X^{\prime}}(m)$, where $O_{X^{\prime}}(1)$ stands for the hyperplane section of $X^{\prime}$ in $P^{n}$.

Proof. Let $Y$ be the affine cone of $X^{\prime}$ via our imbedding and $y$ be its vertex. If we blow up $y$ one get $X^{\prime}$ as exceptional locus. Moreover, $X=V\left(O_{X^{\prime}}(1)\right) \leftrightarrow$ (the vector bundle associated to $\left.O_{X^{\prime}}(1)\right)$ and the immersion $i: X^{\prime} \hookrightarrow X$ (from the diagram $(*)$ ) coincides with the zero section of this bundle. Because $i^{*}(I)=O_{X^{\prime}}(1)$, the corollary follows from proposition 2.

Remark. The use of corollary 2 is that it allows us to see that the vertexes of certain cones are not Gorenstein. For example take as $X^{\prime}$ a non singular complete intersection in $P^{n}$ given by the (homogeneous) equations $f_{1}, \cdots, f_{n-d}$ of degrees $m_{1}, \cdots, m_{n-d}$ respectively. Suppose $d=$ $\operatorname{dim}\left(X^{\prime}\right) \geqslant 3$; then it is well known (see [13]) that $\omega_{X^{\prime}}=O\left(\sum_{i=1}^{n-d} m_{i}-n-1\right)$ and that the Picard group of $X^{\prime}$ is the cyclic (infinite) group generated by the class of $O_{X^{\prime}}(1)$ (see [10], éxposé XII).

Now consider another imbedding of $X^{\prime}$, namely the immersion $h: X^{\prime} \longrightarrow P\left(H^{0}\left(X^{\prime}, O_{X^{\prime}}(s)\right)=P\right.$, where $s$ is a fixed positive integer. Then $h^{*}\left(O_{P}(1)\right)=O_{X^{\prime}}(s)$. The corollary 2 of proposition 2 says therefore that the vertex of the affine cone of $X^{\prime}$ via $h$ is not a Gorenstein point if $\sum_{i=1}^{n-d} m_{i}-n-1$ is not a multiple of $s$. 
THEOREM 2. Let $y \in Y$ be an isolated normal singularity of type $\left(\left(n_{1}, s_{1}\right), \cdots,\left(n_{r}, s_{r}\right)\right)$. Then the local ring $O_{Y, y}$ is Gorenstein if and only if there exists a positive integer a such that $n_{1}+1=a s_{1}, \cdots, n_{r}+1=$ $a s_{r}$ and in this case $\omega_{X}=\varphi^{*}\left(\omega_{Y}\right) \otimes I^{1-a}$.

Proof.*) We have only to prove that $O_{Y, y}$ is Gorenstein, assuming the existence of the integer a such that $n_{1}+1=a s_{1}, \cdots, n_{r}+1=a s_{r}$, because the another part in contained in corollary 1 of proposition 2 . Because $O_{Y, y}$ is Gorenstein if and only if its $m_{y}$-adic completion is so, theorem 1 reduces the problem to the case of the vertex of the the cone considered in the example (see $\S 1$ ). Thus we can work with the diagram $(*)$ in which $Y$ is the projective cone (see EGA II (8.3)) arising from the Segre-Veronese imbedding of type $\left(\left(n_{1}, s_{1}\right), \cdots,\left(n_{r}, s_{r}\right)\right)$, i.e. we can suppose $Y$ projective and nonsingular outside of $y$. The idea of the proof is to prove (according with the theorem $B$ ) that the dualizing sheaf $\omega_{Y}$ is invertible. The main steps are:

Step 1. There exists an open neighbourhood $U$ of $X^{\prime}$ in $X$ such that $\omega_{X} / U=I^{1-a} / U$.

Step 2. There exists an invertible $O_{Y}$-module $\omega$ such that $\omega_{X} \cong$ $\varphi^{*}(\omega) \otimes I^{1-a}$.

Step 3. $\omega$ constructed in step 2 is isomorphic to the dualizing sheaf $\omega_{Y}$.

Proof of step 1. We need the following:

LEMMA. In the notations of the diagram (*) from introduction, assume that $X^{\prime}$ is non singular and $H^{1}\left(X^{\prime}, i^{*} I^{n}\right)=0$ for every $n \geqslant 0$. Then for every invertible $O_{X^{-}}$module having the property $i^{*}(L) \cong O_{X^{\prime}}$, there exists an open neighbourhood $U$ of $X^{\prime}$ in $X$ such that $L / U \cong O_{X} / U$.

Proof of the lemma. We can restrict ourselves to the case where $\varphi(X)=Y$ is affine because the problem is local along $X^{\prime}$. Then the exact sequence

$$
0 \rightarrow I^{n+1} \rightarrow I^{n} \rightarrow i_{*} i^{*}\left(I^{n}\right) \rightarrow 0
$$

gives rise (by tensorizing with $L$ and taking into account that $i^{*}(L)=$ $\left.O_{X^{\prime}}\right)$ to the exact sequence

$$
0 \rightarrow L \otimes I^{n+1} \rightarrow L \otimes I^{n} \rightarrow i_{*} i^{*}\left(I^{n}\right) \rightarrow 0
$$

\footnotetext{
*) See added in proof.
} 
The exact sequence of cohomology and the hypothesis that $H^{1}\left(X^{\prime}, i^{*} I^{n}\right)=0$ for all $n \geqslant 0$ allows us to deduce that the natural homomorphism

$$
H^{1}\left(X, L \otimes I^{n+1}\right) \rightarrow H^{1}\left(X, L \otimes I^{n}\right)
$$

is surjective. Because $Y$ is affine and $\varphi$ is proper, then $H^{1}\left(X, L \otimes I^{n}\right)=0$ for $n$ big enough; thus, by descending induction, one deduces that $H^{1}(X, L \otimes I)=0$. This shows as in the proof of theorem 1 that the homomorphism of restriction $H^{0}(X, L) \rightarrow H^{0}\left(X^{\prime}, O_{X^{\prime}}\right)$ is surjective, therefore there is a section $s \in H^{0}(X, L)$ with the property that $s(x) \neq 0$ for every $x \in X^{\prime}$. Then taking $U=\{x \in X / s(x) \neq 0\}$, the lemma is proved.

In order to prove step 1 , we observe that $\omega_{X}$ is invertible because it is nothing else but the sheaf of differentials of degree $d=$ $\operatorname{dim}(X)$ ( $X$ being non singular). Put $L=\omega_{X} \otimes I^{a-1}$. Then $i^{*}(L)=i^{*}\left(\omega_{X}\right) \otimes$ $i^{*}(I) \otimes i^{*}\left(I^{a-1}\right)=O\left(-n_{1}-1, \cdots,-n_{r}-1\right) \otimes O\left(a s_{1}, \cdots, a s_{r}\right)=O_{X^{\prime}}$. On the other hand the hypothesis of the lemma are fullfiled by $\S 1$, remark 1 . Thus step 1 follows from the lemma.

Proof of step 2. By step 1, $\omega_{X} \otimes I^{a-1}$ is trivial along $X^{\prime}$ and then, by $\S 1$, remark 2 , there exists an invertible $O_{Y}$-module $\omega$ such that $\varphi^{*}(\omega) \cong \omega_{X} \otimes I^{a-1}$, therefore

$$
\omega_{X} \cong \varphi^{*}(\omega) \otimes I^{1-a}
$$

Proof of step 3. Supposing $\omega$ constructed, we show first of all that it gives a duality theorem for invertible $O_{Y}$-modules, i.e. for every invertible $O_{Y}$-module $E$, there is a natural isomorphism between the following (finite dimensional) vector spaces:

$$
H^{p}(Y, E) \cong\left(H^{d-p}(Y, \check{E} \otimes \omega)\right)^{\prime} \quad \text { (dual). }
$$

For, we have $E=\varphi_{*} \varphi^{*}(E)$ and $R^{q} \varphi_{*}\left(\varphi^{*}(E)\right)=0$ for $q>0$ (§1, remark 2). Therefore the Leray spectral sequence

$$
E_{2}^{p q}=H^{p}\left(Y, R^{q} \varphi_{*}\left(\varphi^{*}(E)\right)\right) \Rightarrow H^{p+q}\left(X, \varphi^{*}(E)\right)
$$

degenerates and therefore $H^{p}(Y, E) \cong H^{p}\left(Y, \varphi_{*} \varphi^{*}(E)\right) \cong H^{p}\left(X, \varphi^{*}(E)\right)$

Now, applying the duality theorem on $X$, one get

$$
H^{p}\left(X, \varphi^{*}(E)\right) \cong\left(H^{d-p}\left(X, \varphi^{*}(E)^{\vee} \otimes \omega_{X}\right)\right)^{\prime}
$$

In order to prove (10), one remains to see that there is a natural isomorphism 


$$
H^{d-p}(Y, \check{E} \otimes \omega) \cong H^{d-p}\left(X, \varphi^{*}(E)^{\vee} \otimes \omega_{X}\right) .
$$

Applying once more (11) to $\check{E} \otimes \omega$, one has $H^{d-p}(Y, \check{E} \otimes \omega) \cong H^{d-p}\left(X, \varphi^{*}(\check{E}) \otimes\right.$ $\varphi^{*}(\omega)$ ). Using (9) and this isomorphism, (13) becomes:

$$
H^{d-p}(X, F) \cong H^{d-p}\left(X, F \otimes I^{1-a}\right)
$$

where $F=\varphi^{*}(E) \otimes \varphi^{*}(\omega)$.

First of all observe that $i^{*}(F) \cong O_{X^{\prime}}$. The exact sequence

$$
0 \rightarrow O_{X} \rightarrow I^{-1} \rightarrow i_{*} O\left(-s_{1}, \cdots,-s_{r}\right) \rightarrow 0
$$

tensorized with $F$, gives rise to the exact sequence

$$
0 \rightarrow F \rightarrow F \otimes I^{-1} \rightarrow i_{*} O\left(-s_{1}, \cdots,-s_{r}\right) \rightarrow 0 .
$$

We claim that $H^{q}\left(X^{\prime}, O\left(-s_{1}, \cdots,-s_{r}\right)\right)=0$ for all $q \geqslant 0$. Indeed, all is clear using Künneth's formulas and the fact that $-s_{1}>-n_{1}-1$, $\cdots,-s_{r}>-n_{r}-1$ (because $a s_{\nu}=n_{\nu}+1$ for all $\nu$ )-see also $\S 1$, remark 1. Therefore the long exact sequence of cohomology of (15) shows that $\left(13^{\prime \prime}\right)$

$$
H^{q}(X, F) \cong H^{q}\left(X, F \otimes I^{-1}\right) .
$$

Tensorizing again (14), but now with $F \otimes I^{-1}$, one get the exact sequence

$$
0 \rightarrow F \otimes I^{-1} \rightarrow F \otimes I^{-2} \rightarrow i_{*} O\left(-2 s_{1}, \cdots,-2 s_{r}\right) \rightarrow 0
$$

(because $i^{*}\left(F \otimes I^{-1}\right)=O\left(-s_{1}, \cdots,-s_{r}\right)$ ) and the same remarks as above give :

$$
H^{q}\left(X, F \otimes I^{-1}\right) \cong H^{q}\left(X, F \otimes I^{-2}\right)
$$

and iterating this $(a-1)$-times and taking into account that $(1-a) s_{\nu}>$ $-n_{\nu}-1$ for all $=1,2, \cdots, r$, the formula $\left(13^{\prime}\right)$ is proved.

Now we arrived at the following situation: $Y$ is non singular outside of $y$ and Cohen-Macaulay at $y$ (by corollary 1 of theorem 1 ), $\omega$ is an invertible $O_{Y}$-module which gives a duality theorem for all the invertible $O_{Y}$-modules. If we show that $\omega \cong \omega_{Y}$, theorem 2 will be proved. In order to do this, observe that $\operatorname{dim}_{K} H^{d}(Y, \omega)=1$, because $H^{d}(Y, \omega)=\left(H^{0}\left(Y, O_{Y}\right)\right)^{\prime}=K$ ( $K$ is algebraically closed). Applying the duality theorem $\mathrm{A}$, one get $\operatorname{Hom}_{O_{Y}}\left(\omega, \omega_{Y}\right)=\left(H^{d}(Y, \omega)\right)^{\prime}$ and thus $\operatorname{dim}_{K} \operatorname{Hom}_{O_{Y}}\left(\omega, \omega_{Y}\right)=1$. Let $f \in \operatorname{Hom}_{O_{Y}}\left(\omega, \omega_{Y}\right)$ be a basis of this space; 
one knows that $\omega_{Y}$ is a torsion free $O_{Y}$-module of rank 1 and the canonical map (in which $K(Y)$ is the field of rational functions of $Y$

$$
\operatorname{Hom}_{o_{Y}}\left(\omega, \omega_{Y}\right) \rightarrow \operatorname{Hom}_{K(Y)}\left(\omega \otimes K(Y), \omega_{Y} \otimes K(Y)\right)
$$

is injective (see [1], chapter 1). These imply that the natural homomorphism of restriction

$$
\operatorname{Hom}_{O_{Y}}\left(\omega, \omega_{Y}\right) \rightarrow \operatorname{Hom}_{O_{Y}}\left(\omega / Y-y, \omega_{Y} / Y-y\right)
$$

is also injective, and therefore bijective because the second space is one dimensional. Indeed, this space equals to $H^{0}\left(Y-y, O_{Y} / Y-y\right)$, which is isomorphic to $H^{0}\left(Y, O_{Y}\right)$, because $y$ is a normal point and $\operatorname{dim}(Y) \geqslant 2$. Therefore $f / Y-y$ is an isomorphism. Moreover, $f$ is injective because $\omega$ is invertible and $\omega_{Y}$ is torsion free of rank 1 . Considering the exact sequence

$$
0 \longrightarrow \omega \stackrel{f}{\longrightarrow} \omega_{Y} \longrightarrow G \longrightarrow 0
$$

we saw that $\operatorname{Supp}(G) \subseteq\{y\}$. Finally, we claim that $\chi(Y, \omega)=\chi\left(Y, \omega_{Y}\right)$. For, the duality theorems give: $H^{q}\left(Y, O_{Y}\right)=\left(H^{d-q}(Y, \omega)\right)^{\prime}=\left(H^{d-q}\left(Y, \omega_{Y}\right)\right)^{\prime}$ for every $q \geqslant 0$. Therefore

$$
\chi(Y, \omega)=(-1)^{d} \chi\left(Y, O_{Y}\right)=\chi\left(Y, \omega_{Y}\right) .
$$

The exact sequence (17) and the additivity of the Euler-Poincare characteristic show that $\chi(Y, G)=0$ and therefore $G=0$, because $\operatorname{dim}(\operatorname{Supp}(G)) \leqslant 0$.

The theorem 2 is completely proved.

COROLlaRY 1. Let $y \in Y$ be an isolated normal singularity of type $\left(\left(n_{1}, s_{1}\right), \cdots,\left(n_{r}, s_{r}\right)\right)$. Then $\omega_{Y}$ is invertible in a neighbourhood of $y$ if and only if there exists an integer a (which must be positive) such that $n_{1}+1=a s_{1}, \cdots, n_{r}+1=a s_{r}$. If $a=1$, one has furthermore $\varphi^{*}\left(\omega_{Y}\right) \cong \omega_{X}$.

COROLlaRY 2. The vertex of the affine cone of $P^{n} \times P^{m}$ ( $n$ and $m$ being positive) via the Segre-Veronese imbedding given by forms of bidegree $(s, t)$ is a Gorenstein point if and only if there is an integer a such that $n=$ as and $m=a t$. In particular the vertex of the affine cone of $P^{n} \times P^{m}$ via the Segre immersion is a Gorenstein point if and only if $m=n$. 
Remark. In [9] M. Fiorentini proved (by direct computations, using the implicite equations) that the vertex of the affine cone of the projective line $P^{1}$ via the Veronese imbedding given by forms of degree $s$, is not a Gorenstein point if $s \geqslant 3$. Later, V. Brînzănescu generalized this result to an arbitrary projective space $P^{n}$ (see [8]), showing that the corresponding vertex is Gorenstein if and only if $n+1$ is multiple of $s$. The method used by Brînzănescu is very elementary and works with the parametric equations. These two methods are not more applicable for the Segre imbedding for instance.

Added in proof. 1. The conclusion of theorem 1 can be improved, as G. Pfister observed, in the sense that it remains still true by replacing the completions of $O_{Y, y}$ and $O_{\tilde{Y}, \tilde{y}}$ by their henselizations; this is a simple consequence of theorem 1 and Artin's approximation theory.

2. The proof of theorem 2 can be simplified, using the following characterization: if $\mathrm{Z}$ is a closed subvariety of $P^{n}$, then $Z$ is arithmetically Gorenstein in $P^{n}$ if and only if $Z$ is arithmetically Cohen-Macaulay in $P^{n}$ and the dualizing sheaf $\omega_{Z}$ is isomorphic to $O_{Z}(s)$ for a suitable $s \in Z$. However, the present proof allows us to get a generalization of theorem 2 to the case where $\mathrm{y} \in Y$ is replaced by a suitable subvariety $Y^{\prime} \hookrightarrow Y$, such that this immersion is a Segre-Veronese imbedding (see the forthcoming paper of N. Manolache and the author with this title).

\section{REFERENCES}

[1] A. Altman and S. Kleiman, Introduction to Grothendieck Duality Theory, Springer Lecture Nothes in Math. (1970).

[2] M. Artin, Some numerical criteria for contractibility of curves on an algebraic surface, Amer. J. Math:. 84 (1962), pp. 485-496.

[ 3 ] M. Artin, On isolated rational singularities of surfaces, Amer. J. Math. 88 (1966), pp. 129-136.

[ 4 ] H. Bass, On the ubiquity of Gorenstein rings, Math. Zeit. 82 (1963), pp. 8-28.

[5] L. Bădescu, Contractions rationnelles des variétés algébriques, Annali Sc. Norm. Sup. Pisa, 27 (1973), pp. 743-747.

[6 ] L. Bădescu, Contractions algébriques et applications, Revue Roum. Math. Pur. Appl. XIX, 2 (1974), pp. 143-160.

[7] L. Bădescu et M. Fiorentini, Criteri di semifattorialità e di fattorialità per gli anelli locali con applicazioni geometrche, Annali Mat. Pura Appl. CII (1975), pp. 211-222.

[ 8 ] V. Brînzănescu, Asupra scufundărilor Veronese, Studii Cerc. Matematice Nr. 5 (1974).

[ 9 ] M. Fiorentini, Esempi di anelli di Cohen-Macaulay semifattoriali che non sono di Gorenstein, Rend. Acad. Lincei, series VIII, 50, fasc. 5 (1971). 
[10] A. Grothendieck, Séminaire de Géométrie Algébrique 1962 (SGA II), Paris.

[11] A. Grothendieck, et J. Dieudonné, Éléments de Géométrie Algébrique (E.G.A.), chap. I-III, 1961, Paris.

[12] D. Mumford, Lectures on curves on an algebraic surface, Princeton, New Jersey, 1966.

[13] J. P. Serre, Faisceaux algébriques cohérents (FAC), Annals Math. 61 (1955), p. $197-278$.

University of Bucharest,

Dept. of Mathematics 